\title{
EULERIAN MULTI-PHASE CFD MODEL FOR PREDICTING THE PERFORMANCE OF A CENTRIFUGAL DREDGE PUMP
}

\author{
NICOLÒ BECCATI ${ }^{1}$, CRISTIAN FERRARI $^{1}$, MARCO PARMA ${ }^{2}$ \& MASSIMO SEMPRINI ${ }^{2}$ \\ ${ }^{1}$ CNR - IMAMOTER, Institute for Agricultural and Earthmoving Machines of the Italian National Research \\ Council, Ferrara, Italy. \\ ${ }^{2}$ ITALDRAGHE S.P.A., San Giovanni in Marignano, Italy.
}

\begin{abstract}
Dredge pumps are a complex engineering topic in comparison to water pumps. Mixtures of seawater with several types of soils do not behave as a homogenous fluid, and numerical simulations of these machines can be very challenging. Typical numerical approaches to simulations of dredge pumps are single-phase equivalent slurry and multi-phase liquid-solid, where the specification of the particle flow field can be Eulerian or Lagrangian. The single-phase slurry approach is not sufficient to describe the effects of particle size and concentration of the solid phase on pump performance; for this reason, this paper examines a multi-phase CFD model applied to a dredge pump. The solid phase is modelled with an Eulerian approach, in order to reduce the computational effort required by a Lagrangian method, mainly used for low solid-phase concentrations. The primary purpose of the presented model, developed using commercial software ANSYS CFX, is to predict head losses in a dredge pump working with several particle sizes, from 0.1 to $5 \mathrm{~mm}$, and different volume concentrations of the solid phase, from $20 \%$ to $30 \%$. For numerical solid-phase calibration, the effect of the particle size on pump performance is associated with non-Newtonian rheology of the simulated Eulerian phase. The numerical model is validated via experimental tests on the dredge pump using seawater. The calibration of the particle size effect is obtained from scientific literature data about dredge pump losses in different conditions. The model presented could be a useful tool for the analysis of existing dredge pumps or for the design of new machines.
\end{abstract}

Keywords: centrifugal pumps, CFD, dredge pumps, Eulerian-Eulerian, multi-phase flow.

\section{INTRODUCTION}

Centrifugal pumps are often used to handle large amounts of solid-water slurries, with some decrease in performance due to differences in density and flow path with respect to water [1], similar to the effects of non-Newtonian fluids on the impeller [2-4]. Scientific literature on dredge pump design [5-7] provides many correlations and methods in order to predict and measure pump performance using different solid densities, particle sizes and concentrations, based on empirical studies. Empirical equations can provide a good preliminary study for any design concept of a dredge pump, but modern CAE techniques based on numerical simulations may be a more useful tool, if they are calibrated well on the applications to simulate. For this reason, this paper investigates a CFD model in order to provide a calibration algorithm for a multi-phase dredge pump simulation, validated using experimental tests and literature-based equations.

Slurry centrifugal pumps are often studied with CFD techniques, using different approaches like the single-phase equivalent slurry [8], the Eulerian-Eulerian multi-phase [9] or the Eulerian-Lagrangian multi-phase [10]. Every approach has different pros and cons, depending on the spendable computational effort, the physics numerical calibration and the final goal to achieve. For water-sand slurries with high solid-phase concentrations (> 20\%), the computational fluid dynamics is often studied with an Eulerian-Eulerian approach [11-14], that permits good results without over-demanding computational simulations (Fig. 1). 


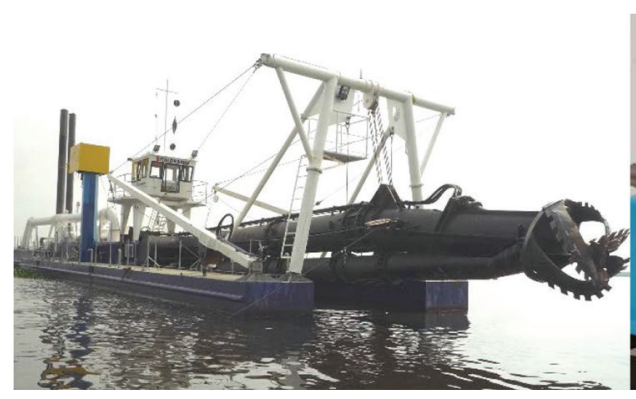

(a)

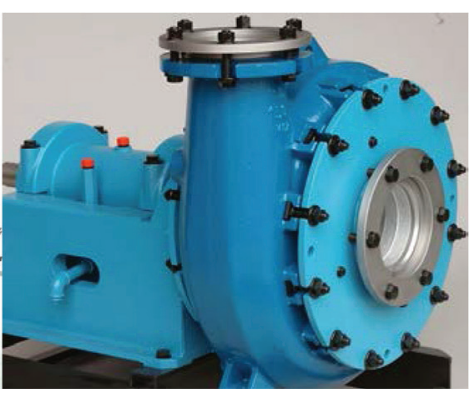

(b)

Figure 1: Examples of dredging machinery: (a) offshore dredger;

(b) dredge pump. Courtesy of Italdraghe S.p.A.

\section{ANALYSED MACHINE}

The analysed machine is a centrifugal dredge pump. The impeller has four blades and the rotational speed available is normally around $1000 \mathrm{rpm}\left(\omega_{0}\right)$. The pump mostly transports solid particles around $25 \%$ volume concentration, with particle diameter sizes in the $0.1-10$ $\mathrm{mm}$ range, depending on the dredging site. The best working efficient point with seawater is $70 \mathrm{~m}$ hydraulic head $\left(\mathrm{H}_{0}\right)$ at $1000 \mathrm{~m}^{3} / \mathrm{s}$ flow rate $\left(\mathrm{Q}_{0}\right)$. The impeller geometry specifications are given in Fig. 2.

\subsection{Experimental tests}

Pump performance was tested on an existing dredging plant, using seawater at $20^{\circ} \mathrm{C}$. The hydraulic head was measured with the outlet-inlet pressure difference with analogue

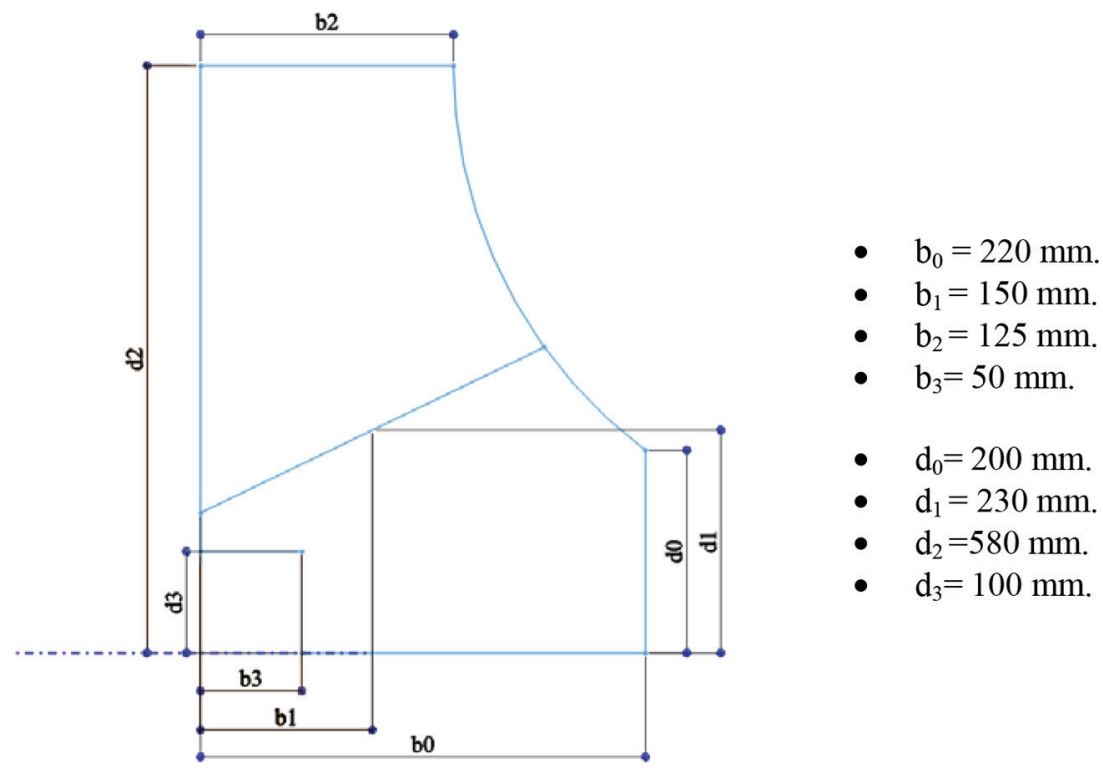

Figure 2: Impeller geometric dimensions. 
Table 1: Experimental operating points with seawater.

\begin{tabular}{lccccccccc}
\hline $\boldsymbol{\omega} / \boldsymbol{\omega}_{\mathbf{0}}$ & & $\mathbf{0 . 6}$ & \multicolumn{3}{c}{$\mathbf{0 . 7}$} & \multicolumn{3}{c}{$\mathbf{0 . 8}$} \\
\hline $\mathrm{H} / \mathrm{H}_{0}$ & 0.31 & 0.29 & 0.27 & 0.38 & 0.36 & 0.31 & 0.52 & 0.49 & 0.44 \\
$\mathrm{Q} / \mathrm{Q}_{0}$ & 0.18 & 0.38 & 0.54 & 0.20 & 0.41 & 0.60 & 0.24 & 0.48 & 0.70 \\
\hline
\end{tabular}

pressure gauges, while the flow rate was measured using an ultrasonic flowmeter on the outlet duct. The rotational pump speed was set by regulating the attached diesel motor power. The measured hydraulic head versus flow rate, by varying the pump rotational speed is given in Table 1.

\section{CFD MODEL}

The simulations are carried out with the commercial CFD code ANSYS CFX, Release 19.2 [15]. The code solves the 3D Cauchy momentum equation using an element-based finitevolume method. The geometry is generated using the commercially available software SOLIDWORKS and then imported into the meshing software ANSYS ICEM, in order to mesh the domain. Then, boundary conditions are imposed on the volume, by defining the multiple frames of reference, the transient analysis parameters and the properties of the materials involved. The multiphase flow simulations largely use the Eulerian-Eulerian approach for the solutions. This approach is based on the principle of interpenetrating continua [16], where the phases share the same volume. The phases, each of which are described by its physical properties and its own velocity, pressure, concentration and temperature field, share the same volume and penetrate each other in space exchanging mass, momentum and energy. For the issue being investigated, the "Volume of Fluid" model was applied, in order to simplify the multiphase simulations and reduce the computational time effort. The simplification performed makes it possible to solve the bulk transport equations that can be derived by summing the individual phasic transport equations for seawater $(w)$ and solid (s):

$$
\begin{gathered}
\rho_{m}=\sum \rho_{i} r_{i} . \\
\bar{U}_{m}=\frac{1}{\rho_{m}} \sum \rho_{i} r_{i} \bar{U}_{i} . \\
\mu_{m}=\sum \mu_{i} r_{i} . \\
i=w, s .
\end{gathered}
$$

The equations describing the mathematical model are mass continuity (4), momentum continuity (5) and volume conservation (6). The energy continuity is not simulated for the isothermal hypothesis applied.

$$
\begin{gathered}
\frac{\partial}{\partial t}\left(\rho_{m} r_{i}\right)+\nabla \cdot\left(\rho_{m} r_{i} \bar{U}_{m}\right)=0 . \\
\frac{\partial}{\partial t}\left(\rho_{m} \bar{U}_{m}\right)+\nabla \cdot\left(\rho_{m} \bar{U}_{m} \otimes \bar{U}_{m}\right)=-\nabla p+\nabla \cdot\left(\mu_{m}\left(\nabla \bar{U}_{m}+\left(\nabla \bar{U}_{m}\right)^{T}\right)\right) .
\end{gathered}
$$




$$
\sum r_{i}=1
$$

These six nonlinear partial differential equations must be solved for the six dependent variables $\left(\mathrm{U}_{\mathrm{mx}}, \mathrm{U}_{\mathrm{my}}, \mathrm{U}_{\mathrm{mz}}, \mathrm{r}_{\mathrm{w}}, \mathrm{r}_{\mathrm{s}}, \mathrm{p}\right)$ [17].

\subsection{Mesh and grid sensitivity}

The pump volume is divided into two finite-volume domains in order to perform a multiple frame of reference simulation: the stationary domain of the volute and a rotational domain of the impeller (see Fig. 3). Between the domains, two interfaces are set with the Mixing Plane model applied as transition from rotor to stator. In this approach, the flow field data are averaged circumferentially from both frames of reference at the interface and passed to the adjacent zone as boundary conditions.

The number of elements is defined after a grid sensitivity analysis, based on the monitoring of the pump hydraulic head. Three grids were generated: mesh 1 , composed of $0.5 \cdot 10^{6}$ elements, mesh 2, composed of $1.3 \cdot 10^{6}$ elements and mesh 3 , composed of $3 \cdot 10^{6}$ elements. In CFD simulations, the numerical results are dependent from the number of elements of the grid used for the simulations. So, as a first step, it is needed a grid sensitivity analysis on the numerical results, in order to find the minimum number of elements that doesn't affect the numerical results. This permits to reach the independence of the numerical results on the number of elements. When CFD simulations involve turbulence, it is needed a very dense grid on the walls, in order to solve correctly the adhesion effect and solve the boundary layer. The inlet and outlet ducts in the volute domain were elongated in order to make the numerical simulations more stable.

\subsection{Boundary conditions}

All the simulations are carried out as steady-state analysis. The multi-phase model is set as homogeneous, calculating a unique flow field for the materials involved. The hydraulic head versus flow rate curves are calculated by varying the flow rate developed by the pump. The flow rate value is imposed on the outlet surface of the volute, while the inlet surface is set as a constant relative pressure condition. The solid-phase concentration inside the domains is set as the initial overall condition on the simulated volume and on the outlet flow rate. All the walls are set as smooth walls, with a No Slip condition applied (Table 2).

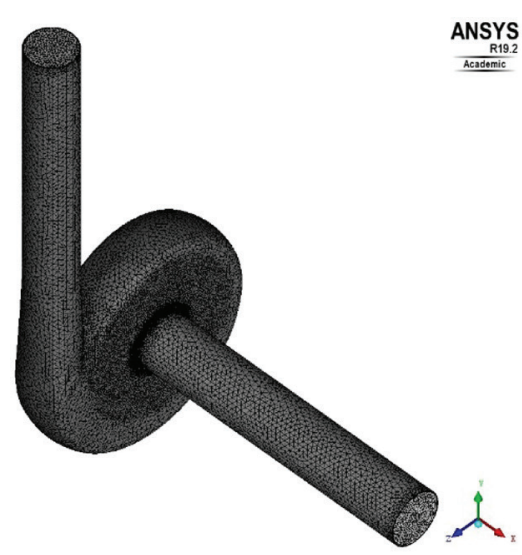

(a)

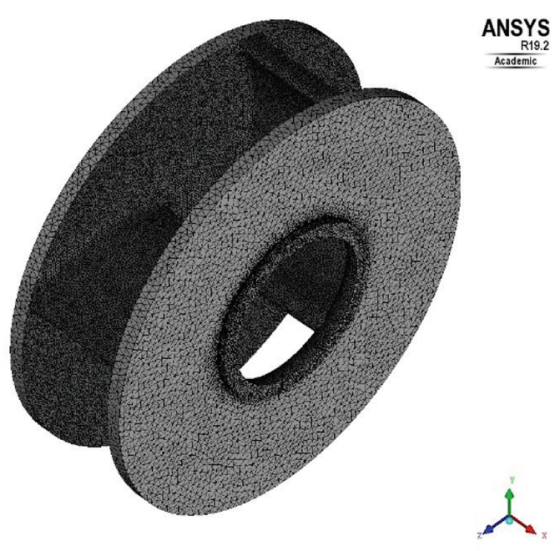

(b)

Figure 3: 3D mesh of the dredge pump domains: (a) volute and (b) impeller. 
Table 2: Simulation settings applied.

\begin{tabular}{llll}
\hline Setting & Value & Setting & Value \\
\hline Seawater density & $1025 \mathrm{~kg} / \mathrm{m}^{3}$ & Solid density & $2660 \mathrm{~kg} / \mathrm{m}^{3}$ \\
Advection scheme & High Resolution & Turbulence model & $\kappa-\omega \mathrm{SST}$ \\
Inlet rel. pressure & $0 \mathrm{bar}$ & Outlet flow rate & $0-3 \mathrm{Q}_{0}$ \\
\hline
\end{tabular}

\subsection{Seawater simulation results}

The validation of the numerical model is reached with single-phase simulations with seawater: once the numerical convergence is reached, with residual criteria set at $10^{-4}$, the hydraulic head, which varies with the flow rate and the rotational speed, is compared with the experimental results. Figures 4 and 5 show a good match between the numerical and the experimental results; the numerical pump model is then validated.

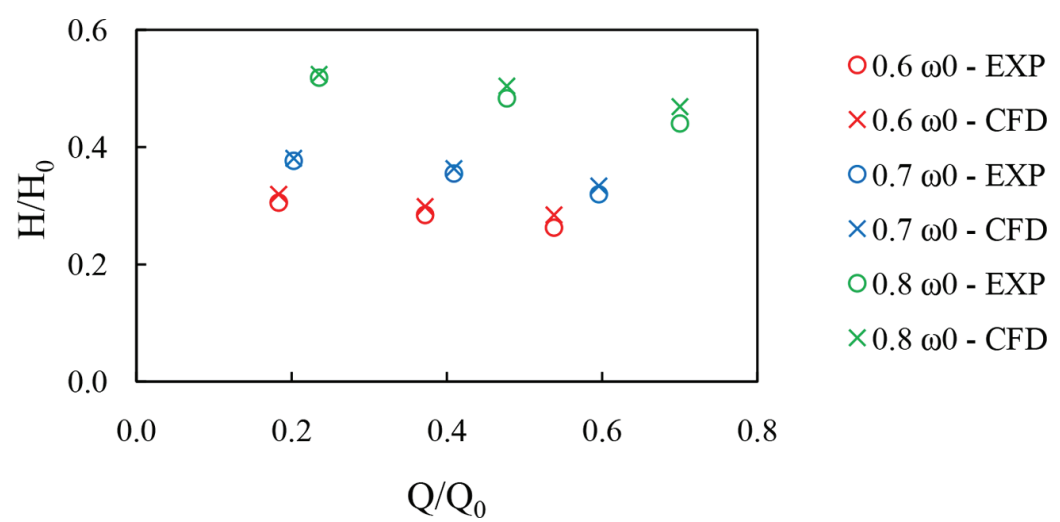

Figure 4: Comparison between experimental and numerical data with seawater.

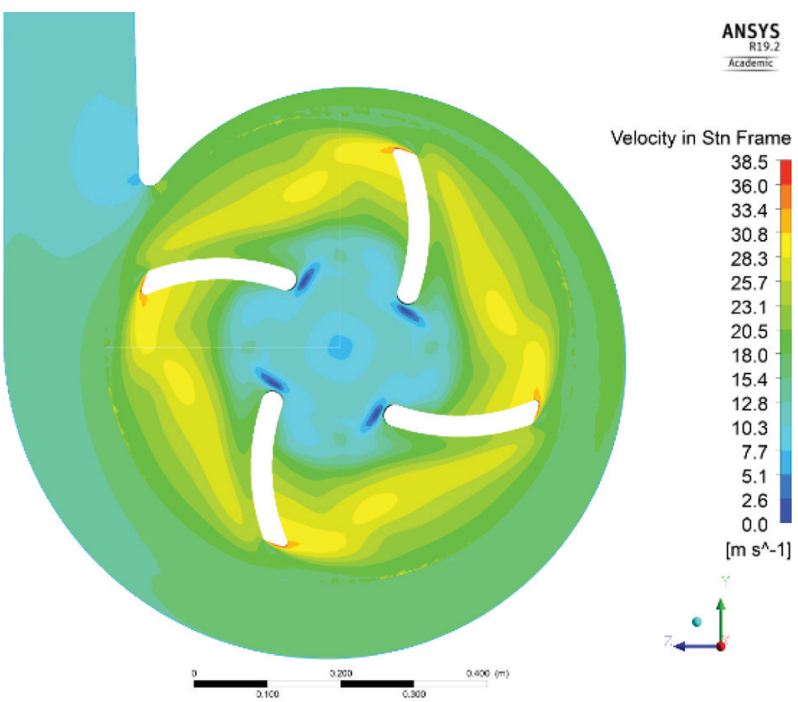

(a) 


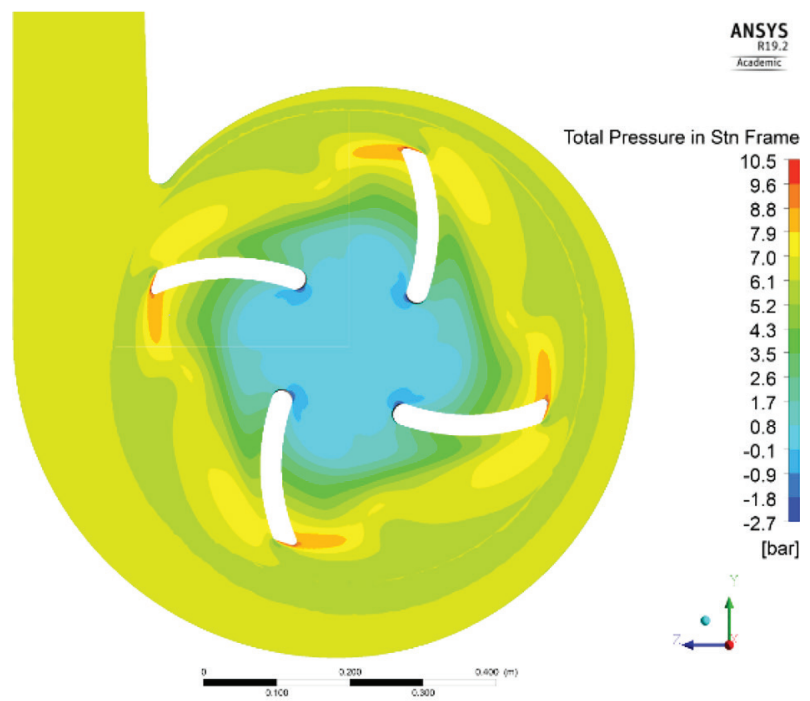

(b)

Figure 5: YZ middle-plane contours with seawater: (a) velocity and (b) total pressure.

\section{NUMERICAL CALIBRATION}

The rheological models that better describe the behaviour of a water-sand suspension like the one presented here are the non-Newtonian models, like the Bingham model [18], the Ostwaldde Waele model [19] and the Herschel-Bulkley model [20-22]. The last one is the model used for the calibration of the Eulerian solid-phase rheology: $\tau=\tau_{o}+\mathrm{K} \dot{\gamma}^{n}$. (7)

The Herschel-Bulkley model is set on the numerical viscosity properties of the solid phase, in order to match a hydraulic head reduction effect of the non-Newtonian viscosity [23] to the prediction of the head degradation due to the solid effect [24]. While the power index value can be related to the concentration of the solid phase [22], the effect of the particle size is related here to the yield stress value of the viscosity model. The calibration of the yield stress is performed through a sensitivity analysis of the expected head reduction at the fixed particle size with the numerical results by varying the yield stress value on the pump. The prediction of the effect of the particle size on the hydraulic head reduction is calculated with the $K$-factor value, taking into account the effect of the pump radius [3].

For the calibration analysis, the flow rate developed was set constant at $\mathrm{Q}_{0}$ value, while the solid concentration was set at $20 \%$ and $30 \%$. For every particle size, the resulting yield stresses at different solid concentrations were mediated, in order to extract a unique value of the yield stress. The calibration curve of the yield stress versus particle size is provided in Fig. 6; the logarithmic trend is used as the formulation in the numerical yield stress setting, following eqn (8). $\tau_{0}=931.8 \ln (\theta)+7540$. (8)

The plastic viscosity value, related to the solid concentration [7], is given by the Thomas eqn (9):

$$
K=\mu_{w}\left(1+2.5 r_{s}+10.05 r_{s}^{2}+0.00273 \exp \left(16.6 r_{s}\right)\right.
$$




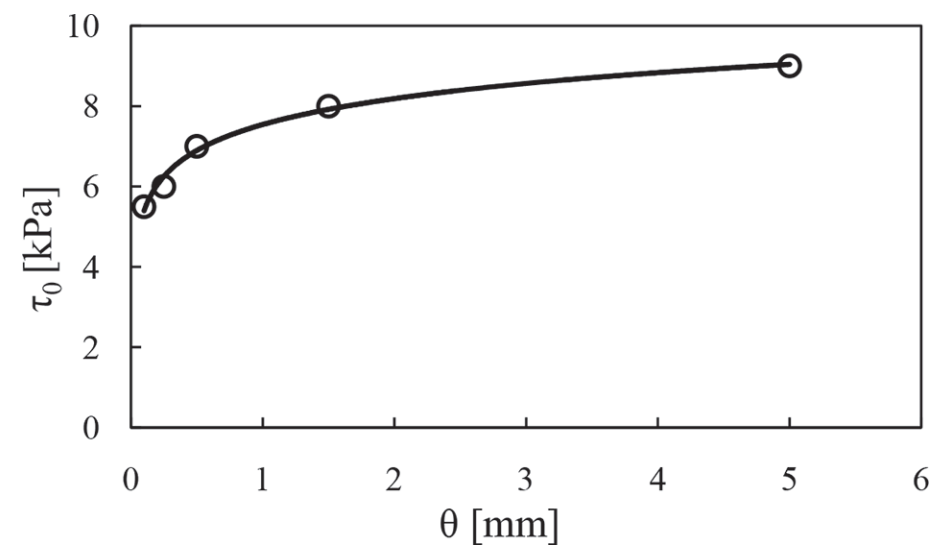

Figure 6: Logarithmic trend curve of yield stress versus particle size.

\section{RESULTS}

The simulations were carried out at five values of particle size, between 0.1 and $5 \mathrm{~mm}$, two values of solid concentrations, $20 \%$ and $30 \%$, and seven values of flow rate at constant speed $\omega_{0}$ (Figs. 7 and 8). The performance curves of hydraulic head versus bulk flow rate were given with a cubic trend line on the resulting points. The hydraulic head was calculated with the following eqn:

$$
H=\Delta p / \rho_{m} g
$$

The results show a hydraulic head reduction, related to seawater, up to $30 \%$ developing $5 \mathrm{~mm}$ solid size at $30 \%$ volume concentration (Fig. 9). The decreasing performance matches the predictions made with the empirical formulae used for calibration at the larger particle

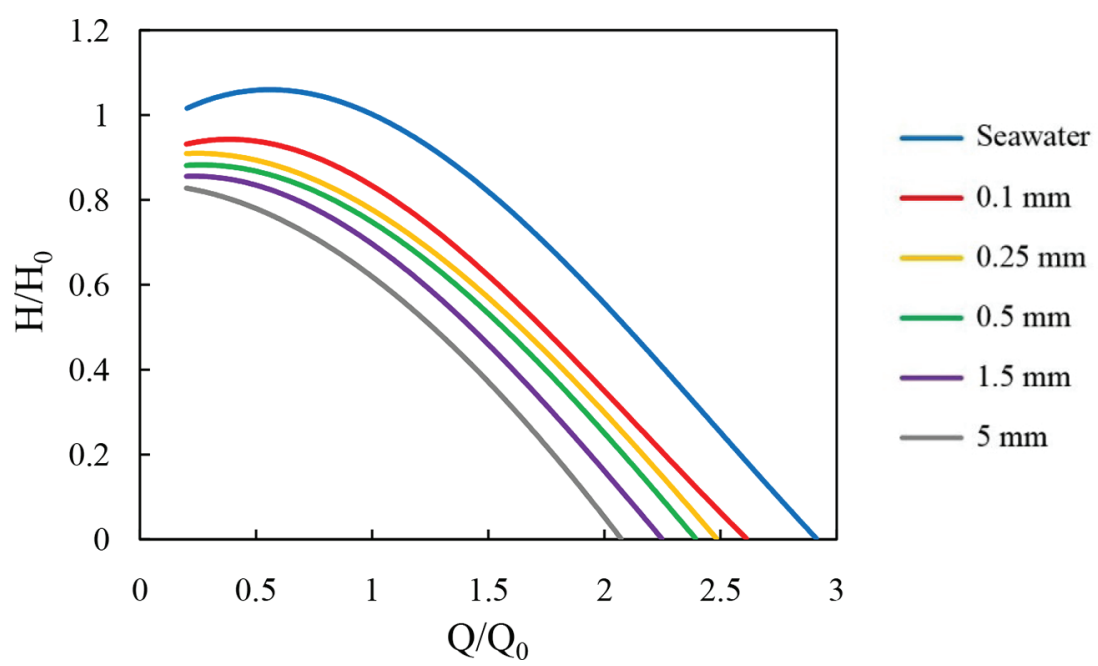

Figure 7: Numerical bi-phase curves with $20 \%$ of solid. 


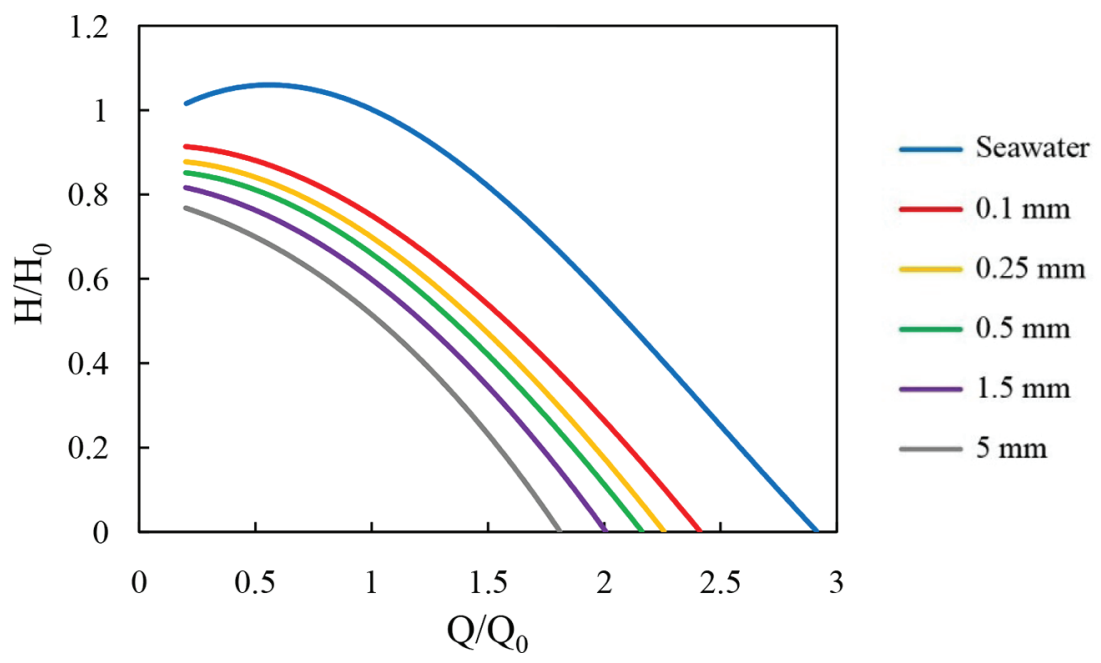

Figure 8: Numerical bi-phase curves with $30 \%$ of solid.

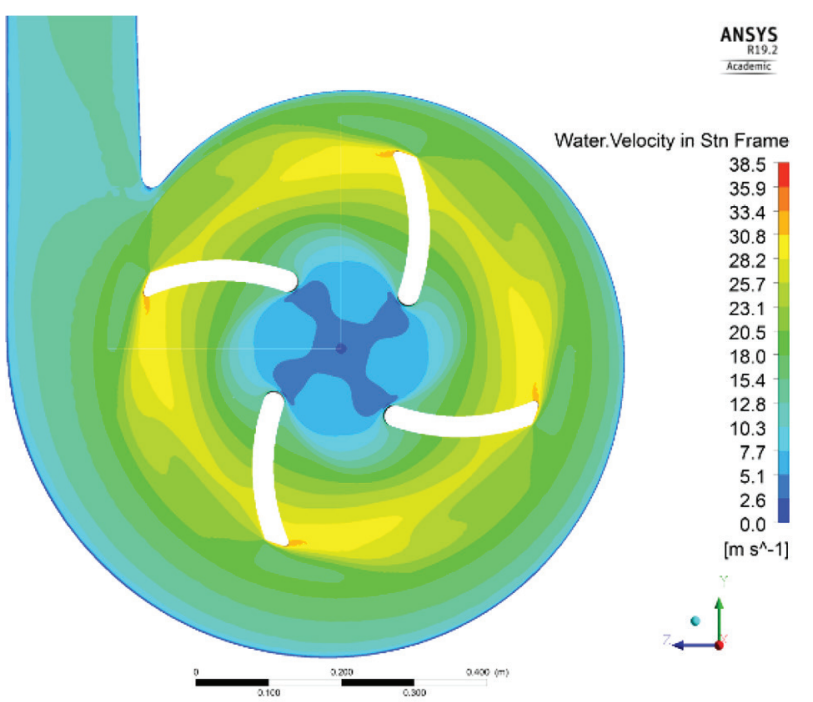

(a) 


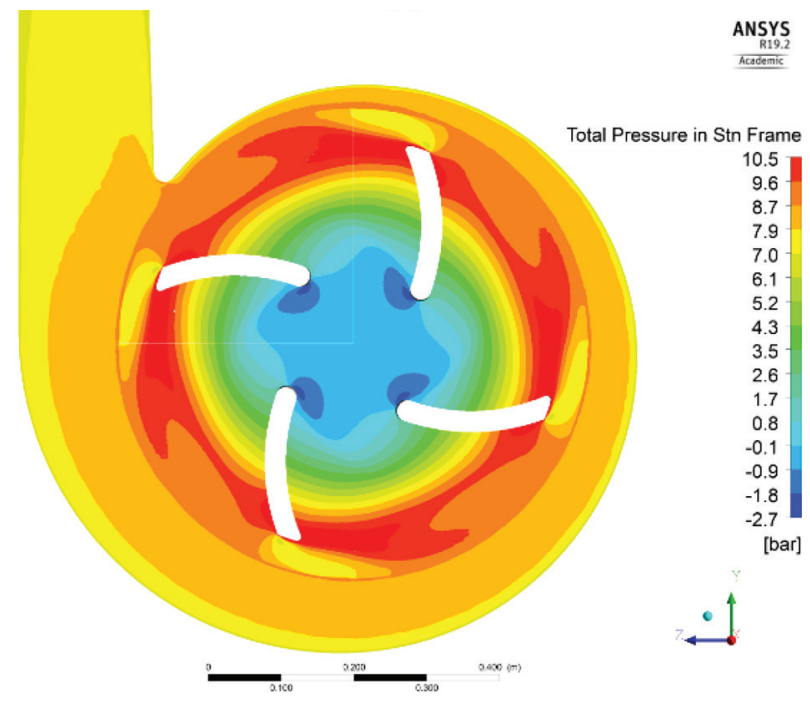

(b)

Figure 9: YZ middle-plane contours with $30 \%$ concentration of $5 \mathrm{~mm}$ solid particles: (a) velocity and (b) total pressure.

sizes. The greatest deviation from the hydraulic head reduction predictions is present at the lower particle sizes, where the numerical results show a 5-10\% decrease, while the empirical formulae show $2-3 \%$.

\section{CONCLUSION}

This paper presents a multi-phase CFD model applied to a dredge pump. The slurry is modelled with a two-phase solid-water mixture using an Eulerian-Eulerian approach, in order to better compute the high solid-phase concentrations involved. The numerical model is developed in order to predict the hydraulic head losses at different particle sizes, from 0.1 to $5 \mathrm{~mm}$, using a non-Newtonian viscosity model applied to the Eulerian solid phase. The validation is performed using experimental test performance levels with seawater, while the calibration of the rheology parameters was performed using scientific literature criteria. The results show a good agreement on the hydraulic losses at higher transported particle sizes, while the greatest deviation is reached at lower particle sizes. Using fast simulations, the presented model can analyse the performance levels of existing dredge pumps or predict losses in new dredge pump concepts. Further analysis and improvements on the presented model can be made, for example, by changing the geometry, empirical formulations for loss predictions or exploring other non-Newtonian models for the solid-phase numerical properties.

\section{ACKNOWLEDGEMENTS}

We would like to thank Italdraghe S.p.A. for the 3D CAD of the pump and the experimental performance values courtesy. 
NOMENCLATURE

\begin{tabular}{llll}
\hline Variable & Description & Variable & Description \\
\hline$\omega_{0}$ & Reference rotational speed & $H_{0}$ & Best efficiency point hydraulic head \\
$Q_{0}$ & $\begin{array}{l}\text { Bulk volume flow rate at } \\
\text { best efficiency point }\end{array}$ & $b$ & Axial length \\
$d$ & Diameter & $\omega$ & Rotational speed \\
$H$ & Hydraulic head & $Q$ & Bulk volume flow rate \\
$\rho$ & Density & $r$ & Volume fraction \\
$\bar{U}$ & Flow velocity & $\mu$ & Dynamic viscosity \\
$p$ & Pressure & $\tau$ & Shear stress \\
$\tau_{0}$ & Yield stress & $K$ & Plastic viscosity \\
$\dot{\gamma}$ & Shear rate & $n$ & Power index \\
$\theta$ & Particle size & $g$ & Standard gravity \\
\hline
\end{tabular}

\section{REFERENCES}

[1] Gülich, J.F., Centrifugal Pumps, 3rd edn., Springer-Verlag Berlin Heidelberg, pp. 929-40, 2014.

[2] Karassik, I.J., Messina, J.P., Cooper, P. \& Heald, C.C., Pump Handbook, 4th ed., The McGraw-Hill Companies, Inc., 2008.

[3] Wilson, K.C., Addie, G.R., Sellgren, A. \& Clift, R., Slurry Transport Using Centrifugal Pumps, Springer Science+Business Media, Inc., 2006.

[4] Buratto, C., Occari, M., Aldi, N., Casari, N., Pinelli, M., Spina, P.R. \& Suman, A., Centrifugal pumps performance estimation with non-Newtonian fluids: review and critical analysis, Proceedings of 12th European Conference on Turbomachinery Fluid dynamics \& Thermodynamics, pp. 1-13, 2017.

[5] Herbich, J.B., Handbook of Dredging Engineering, 2nd ed., McGraw-Hill Professional, 2000.

[6] Vlasblom, J.W., Dredge Pumps, Delft University of Technology Lecture notes, 2004.

[7] Miedema, S.A. \& Ramsdell, R.C., Slurry Transport: Fundamentals, A Historical Overview \& The Delft Head Loss \& Limit Deposit Velocity Framework, Delft University of Technology, 2016.

[8] Ye, D.X., Li, H., Zou, C.H. \& Jiang, B., Characterization of the fluidization of medium consistency pulp suspensions in a centrifugal pump, WIT Transactions on Engineering Sciences, 89, pp. 67-76, 2015.

[9] Pagalthivarthi, K.V., Gupta, P.K., Tyagi, V. \& Ravi, M.R., CFD predictions of dense slurry flow in centrifugal pump casings. International Journal of Mechanical and Mechatronics Engineering, 5(3), pp. 538-550, 2011.

[10] Shen, Z., Chu, W., Li, X. \& Dong, W., Sediment Erosion in the Impeller of a Doublesuction Centrifugal Pump-A case study of the Jingtai Yellow River Irrigation Project, China, Wear. 
[11] Nabil, T., El-Sawaf, I. \& El-Nahhas, K., Sand-water slurry flow modelling in a horizontal pipeline by computational fluid dynamics technique, Int. Water Technol. J, 4(1), pp. 1-17, 2014.

[12] Wanker, R., Goekler, G. \& Knoblauch, H., Numerical modeling of sedimentation utilizing a Euler/Euler approach, WIT Transactions on Engineering Sciences, 30, pp. 327-336, 2001.

[13] Yang, J. \& Chalaturnyk, R.J., Computational fluid dynamics simulation of a very dense liquid-solid flow using a Eulerian model. WIT Transactions on Engineering Sciences, 50, pp. 305-314, 2005.

[14] Tebowei, R., Hossain, M., Oluyemi, G. \& Islam, S., Modelling effects of particle size and pipe gradient on sand transport in multiphase pipes. WIT Transactions on Engineering Sciences, 89, pp. 323-334, 2015.

[15] ANSYS, Inc., ANSYS CFX-Solver Theory Guide, Release 19.2, 2018.

[16] Enwald, H., Peirano, E. \& Almstedt, A.E., Eulerian two-phase flow theory applied to fluidization. Int. J. Multiphase Flow, 22, pp. 21-66, 1996.

[17] Hiltunen, K., Jäsberg, A., Kallio, S., Karema, H., Kataja, M., Koponen, A., Manninen, M. \& Taivassalo, V., Multiphase Flow Dynamics Theory and Numerics, VTT Publications 722, Edita Prima Oy, Helsinki, 2009.

[18] Shakibaeinia, A. \& Jin, Y.C., Lagrangian multiphase modeling of sand discharge into still water, Advances in Water Resources, 48, pp. 55-67, 2012.

[19] Konijn, B.J., Sanderink, O.B.J. \& Kruyt, N.P., Experimental study of the viscosity of suspensions: Effect of solid fraction, particle size and suspending liquid, Powder Technology, 266, pp. 61-69, 2014.

[20] Olhero, S.M., Ferreira \& J.M.F., Influence of particle size distribution on rheology and particle packing of silica-based suspensions, Powder Technology, 139, pp. 69-75, 2004.

[21] Mueller, S., Llewellin, E.W. \& Mader, H.M., The rheology of suspensions of solid particles, Proceedings of the Royal Society A: Mathematical, Physical and Engineering Sciences, 466(2116), pp. 1201-1228, 2010.

[22] Mangesana, N., Chikuku, R.S., Mainza, A.N., Govender, I., van der Westhuizen, A.P. \& Narashima, M., The effect of particle sizes and solids concentration on the rheology of silica sand based suspensions. The Journal of The Southern African Institute of Mining and Metallurgy, 108, pp. 237-243, 2008.

[23] Li, W.G., Effects of viscosity of fluids on centrifugal pump performance and flow pattern in the impeller. International Journal of Heat and Fluid Flow, 21(2), pp. 207-12, 2000.

[24] Engin, T. \& Gur, M., Comparative evaluation of some existing correlations to predict head degradation of centrifugal slurry pumps. ASME Journal of fluids engineering, 125(1), pp. 149-57, 2003. 